\title{
Long-term adherence to CPAP treatment in patients with obstructive sleep apnea: importance of educational program
}

This article was published in the following Dove Press journal:

Patient Preference and Adherence

3 November 20II

Number of times this article has been viewed

\author{
Giuseppe Emanuele La Piana' \\ Alessandro Scartabellati' \\ Lodovico Chiesa' \\ Luca Ronchi' \\ Paola Raimondi' \\ Miriam A Carro' \\ Silvia Zibetti' \\ Stefano Aiolfi \\ 'Pulmonary Rehabilitation Unit, \\ S. Marta Hospital, Rivolta D'Adda; \\ ${ }^{2}$ Unit of Pneumology, \\ AO Ospedale Maggiore di Crema, \\ Crema, Italy
}

Background: Lack of adherence with continuous positive airway pressure (CPAP) therapy is the major cause of treatment failure in patients with obstructive sleep apnea syndrome. We evaluated the effectiveness of our intensive educational program on adherence in the short term and the long term.

Methods: The educational program consisted of: intensive training, whereby each patient performed individual and collective sessions of three hours receiving information about obstructive sleep apnea syndrome, familiarizing themselves with CPAP tools, on six consecutive days; long-term training; and support meetings, with reassessment at three months and one year. Results: In 202 patients with obstructive sleep apnea syndrome, the mean (standard deviation) apnea/hypopnea index was $45 \pm 22$, the Epworth Sleepiness Scale score was $14 \pm 5$, and the average titration pressure was $10 \pm 2 \mathrm{~cm} \mathrm{H}_{2} \mathrm{O}$. At three months, 166 patients (82\%) used CPAP for an average of 7.3 hours per night. At one year, 162 (80\%) used CPAP for about seven hours per night. At two years, 92 patients (43\%) used CPAP for about five hours per night. The level of satisfaction remained higher in patients in ventilation.

Conclusion: Our data show strong adherence to CPAP at three months and one year, with a decrease at two years. The initial educational program seems to play an important role in adherence. This effect is lost in the long term, suggesting that periodic reinforcement of educational support would be helpful.

Keywords: adherence, continuous positive airway pressure, obstructive sleep apnea syndrome, educational program

\section{Introduction}

Obstructive sleep apnea syndrome is a disorder characterized by repeated interruption of breathing ${ }^{1}$ due to obstruction of the upper airway. ${ }^{2}$ The prevalence of obstructive sleep apnea syndrome is $4 \%-5 \%$ in the general population, and is associated with increased cardiovascular risk, ${ }^{3}$ increased risk of car accidents, ${ }^{4}$ and increased use of health care resources. ${ }^{5}$ Continuous positive airway pressure (CPAP) therapy for obstructive sleep apnea syndrome reduces daytime sleepiness, ${ }^{6}$ improves cognitive function, ${ }^{7}$ and reduces sympathetic neural activation, blood pressure, ${ }^{8}$ and mortality. These results are related to proper adherence with treatment. ${ }^{10}$ Side effects, such as claustrophobia, difficulty in using the machine, and psychological or psychosocial factors, can affect night-time ventilator use. ${ }^{11}$

Predictors of adherence to night-time CPAP treatment for obstructive sleep apnea are still lacking. ${ }^{12}$ Suspension of CPAP use restores symptoms and the risks of obstructive sleep apnea at critical levels. ${ }^{13}$ It is estimated that between $29 \%$ and $83 \%$ of patients 
are not adherent with CPAP ventilation. The most recent definition of CPAP adherence is ventilator use for at least four hours per night on at least $70 \%$ of nights, and for at least 30 days in the first three months after prescription. ${ }^{14}$ It is stressed that the main problem with treatment of obstructive sleep apnea syndrome is poor adherence to CPAP in the short term, and even more so in the long term. ${ }^{15}$

Various methods have been proposed to increase compliance, such as achieving the proper titration, using the most appropriate ventilator, eg, auto-CPAP, auto-BiPAP (bilevel positive airway pressure), proper humidification of the upper airways, and educational and psychological programs. ${ }^{11}$ These interventions are based on theoretical models, and their aim is to remove all barriers that the patient perceives in using CPAP. This suggests the need for an educational program, as recommended in the literature, ${ }^{16-18}$ with structured models developed in hospital.

The role of education in improving CPAP use in the long term has only received modest attention until now. Likar and al showed that therapeutic education of two hours every six months increases CPAP use by at least one hour per night in more than $90 \%$ of patients. ${ }^{19}$ In this study, we assessed the educational effectiveness of an educational protocol for adherence to CPAP at three months and one year after titration, and its effect was still present at two years. Our educational program comprised two stages, ie, initial "intensive" training and a subsequent "periodic" follow-up approach.

\section{Methods}

Two hundred and two patients diagnosed with obstructive sleep apnea were recruited consecutively from the Pulmonary Rehabilitation Unit at Rivolta d'Adda Hospital from January 1, 2004 to May 31, 2008. Inclusion criteria were age 25-85 years, a new polysomnographic diagnosis of obstructive sleep apnea, and not having received previous treatment with noninvasive mechanical ventilation. The severity of obstructive sleep apnea was assessed by the apnea-hypopnea index (AHI, ie, the number of apneas and hypopneas per hour).

Apneas and hypopneas were analyzed according to the guidelines of the American Academy of Sleep Medicine Task Force ${ }^{20}$ with apnea defined as absence of air flow for at least 10 seconds and hypopnea as a reduction in air flow of at least $30 \%$ associated with desaturation of $4 \%$. An AHI of at least 15 events per hour was considered the lower limit for enrolment. The anthropometric characteristics and baseline measures of disease severity for the study population are shown in Table 1.
Table I Baseline characteristics and anthropometric measures of disease severity in the study population

\begin{tabular}{ll}
\hline Gender, M/F & $159 / 43$ \\
Age (years) & $57 \pm 12$ \\
BMI $\left(\mathrm{kg} / \mathrm{m}^{2}\right)$ & $36 \pm 8$ \\
$\mathrm{AHI}($ events/hour) & $45 \pm 22$ \\
$\mathrm{TSpO}<90 \%$ & $14 \pm 18$ \\
ESS & $14 \pm 5$ \\
CPAP average $\left(\mathrm{cm} \mathrm{H}_{2} \mathrm{O}\right)$ & $10 \pm 2$ \\
\hline
\end{tabular}

Note: Values are expressed as the mean \pm standard deviation.

Abbreviations: AHI, apnea-hypopnea index per hour; CPAP, continuous positive airway pressure; ESS, Epworth Sleepiness Scale; $\mathrm{TSpO}_{2}<90 \%$ percentage of recording time spent with saturation below $90 \%$.

All patients underwent an adaptive training program in two steps, comprising "intensive" adaptive training for three hours on six consecutive days after CPAP titration, using an individually tailored technical and practical approach, ${ }^{21}$ followed by periodic follow-up in a group setting to reinforce the concepts and treatment techniques relevant to obstructive sleep apnea. ${ }^{21}$ The study protocol was approved by the local ethics committee.

During the six days of intensive training, each patient underwent both individual and group training. The follow-up support program provided for an ambulatory meeting at three months and one year. At three months, one year, and two years, hours of adherence and degree of satisfaction were evaluated on a subjective three-level scale (poor, enough, good) by each patient. Hours of CPAP adherence were assessed by the memory card in the home ventilator. Adherence to CPAP treatment was defined as a minimal acceptable duration of use of at least four hours per night on $>70 \%$ of nights monitored. Subjects who spontaneously suspended CPAP or were absent from the scheduled control checks, were considered to be nonadherent.

\section{Statistical analysis}

Descriptive statistics were used to describe the study population. Statistical analysis was performed using the Stat View software package (StatView, SAS Institute Inc, Cary, NC). A nonparametric method Mann-Whitney test was performed. A $P$ value less than 0.05 was considered to be statistically significant.

\section{Intensive adaptive training}

The following steps were performed strictly according to the order stated and were repeated several times depending on the patient's subjective response (see Figure 1). 


\begin{tabular}{|l|l|l|}
\hline & Aims & Interventions \\
\hline Step 1 & To provide basic information on OSAS & Verbal approach supported by leaflets \\
\hline Step 2 & To familiarize patient with CPAP & $\begin{array}{l}\text { Practical demonstration on use of CPAP ventilator } \\
\text { Simulation of concrete situations with ventilator use }\end{array}$ \\
\hline Step 3 & $\begin{array}{l}\text { To understand different comfort levels and } \\
\text { features of different masks }\end{array}$ & To test different models and different masks \\
\hline Step 4 & $\begin{array}{l}\text { To prepare patient for use of night CPAP } \\
\text { in long term }\end{array}$ & $\begin{array}{l}\text { To interview patients to provide solutions to any } \\
\text { problems with CPAP use }\end{array}$ \\
\hline
\end{tabular}

Figure I Intensive training.

Abbreviations: CPAP, continuous positive airway pressure; OSAS, obstructive sleep apnea syndrome.

\section{Step I}

The aim of the first step was to provide basic information on obstructive sleep apnea, focusing on a proper knowledge of the etiology of sleep apnea, how it occurs, what causes it, the health risks involved if it is left untreated, and the treatments available, in order to improve patient awareness for the illness. A verbal approach was taken, based on a brochure (Figure 3) given to patients, in which the use of CPAP use was explained.

\section{Step 2}

The aims of the second step were to enable the patient to become familiar with the equipment used for home CPAP, to improve awareness of the CPAP circuitry and of each accessory (whisper, tubing, filters), and to provide a modality of maintenance and regular cleaning of the machine, with a telephone number to call in the event of machine failure. This was achieved by practical demonstration and simulation of ventilator use, and of the alarm and humidification systems (Figure 4).

\section{Step 3}

The purpose of the third step was to introduce the features and comfort of the different nasal masks and caps, and to provide solutions to problems with nasal masks, in particular the possible impact of claustrophobia. This step involved performing tests with different models and different masks.

\section{Step 4}

The final step was to prepare the patient for use of CPAP in the long term and to provide solutions to problems with the home ventilator (Figure 5). This step comprised a question and answer session, directed to build trust and collaboration in the patient/caregiver relationship.

\section{Long-term support program}

The long-term support program (Figures 2 and 6) was based on educational criteria, and had the following components:

- Coordination of training groups according to homogeneity of patients

- Issuing of subsequent clinical appointments

- Chaired group sessions with frontal teaching, coordinated by professional figures (neurophysiopathology technician, physiotherapist, and nurse), strengthening concepts and behaviors learned in intensive training, and to encourage interindividual comparisons of common issues for patients

\begin{tabular}{|c|c|c|}
\hline & Training group & Personalized interventions \\
\hline $\begin{array}{l}\text { Scheduled } \\
\text { meetings } \\
\text { according to a } \\
\text { fixed timetable }\end{array}$ & $\begin{array}{l}\text { To form groups according to criteria of } \\
\text { homogeneity of patients } \\
\text { Group sessions coordinated by physiotherapist and } \\
\text { nurse to reinforce concepts learned in intensive } \\
\text { training and to stimulate dialog on common } \\
\text { concerns of patients }\end{array}$ & $\begin{array}{l}\text { To collect data on parameters of clinical follow-up of } \\
\text { each patient } \\
\text { To read CPAP counter hours to evaluate actual use of } \\
\text { CPAP } \\
\text { To decide with physician the next polysomnographic } \\
\text { controls for re-evaluating the effective CPAP pressure } \\
\text { needed }\end{array}$ \\
\hline
\end{tabular}

Figure 2 Long-term training.

Abbreviation: CPAP, continuous positive airway pressure. 


\author{
When to use CPAP \\ To be effective, treatment should always be used when sleeping \\ This also applies during the afternoon nap \\ Face mask should remain on for duration of sleep \\ When you fall asleep, do not forget to activate the ramp of the device for CPAP ventilation; the ramp function is \\ used to deliver the pressure slowly over 20 minutes before reaching the preset value, so you can sleep without \\ being disturbed by the device pressure delivered \\ If you wake up and you take off the mask, you must return it to the face and re-enable the ramp before sleep.

\section{Benefits of CPAP} \\ There are many reasons to use CPAP: \\ You will feel less tired, less sleepy, and full of energy \\ Risk of cardiovascular and cerebrovascular diseases, such as hypertension, heart attack, and stroke, will decrease.

\section{Any problems with use of CPAP} \\ Call the center that has prescribed CPAP \\ Most problems can be easily resolved \\ If the device does not work call the manufacturers \\ If you break the mask or a component of the circuit, refer to the center that has prescribed CPAP \\ In any case, do not stop using CPAP without contacting the reference center.
}

Figure 3 Brochure on guidelines for CPAP use given to patient.

Abbreviation: CPAP, continuous positive airway pressure.

- Collection of data for clinical follow-up on: body mass index for evaluation of weight loss; Epworth Sleepiness Scale score for evaluation of daytime sleepiness; and actual CPAP use assessed using the CPAP counter

- Establishing a control polysomnographic pressure with the physician to enable re-evaluation of any new effective CPAP titration.

\section{Results}

All 202 patients with obstructive sleep apnea enrolled in this study completed their intensive adaptive training and started CPAP home ventilation. The main anthropometric characteristics and polysomnographic data for the study population are summarized in Table 1 . The patients comprised 159 males and 43 females, with a mean age of $57 \pm 12$ years, body mass index of $36 \pm 8$, AHI of $45 \pm 22$ events per hour, and an Epworth Sleepiness Scale score of $14 \pm 5$. Three months after the beginning of ventilation (average pressure $10 \pm 2 \mathrm{~cm} \mathrm{H}_{2} \mathrm{O}$ ), CPAP adherence was $82 \%$. Ten patients decided to terminate CPAP treatment (in this group basal Epworth Sleepiness Scale was $12 \pm 3$ ) and 26 were lost to follow-up. Adherence to CPAP was $80 \%$ one year after the long-term support program. Four patients decided to terminate CPAP treatment. Two years after the start of treatment and at one year since the last session of the support program, long-term adherence to ventilation was $43 \%$. Seventy-eight patients decided to terminate CPAP treatment (in this group basal Epworth

- Try to wear a mask (out of circuit) while awake for one hour per day

- Try to breathe through the mask (connected to the circuit) keeping your mouth closed, breathing while watching television, or reading the newspaper, awake for an hour per day

- Try to use CPAP during the afternoon nap

- Try to use CPAP during the first 3-4 hours of night sleeping

- Try to use CPAP all night

Go to the next step every two days, once you can wear the mask without anxiety.

Figure 4 Approach to CPAP therapy in the event of claustrophobia. Abbreviation: CPAP, continuous positive airway pressure. 
In the event of claustrophobia, gradual steps are recommended (Figure 4)

\section{Loss of humidification}

Because of CPAP, the inhaled air dries and extra humidification is required.

In most cases, a humidifier system is used at the beginning of the CPAP circuit, but its effectiveness is limited and less than a humidifier or heater. Occasionally, excessive humidity can irritate the nasal mucosa and sinuses and the air temperature may be excessive. Personalized adjustments are recommended and should prevent the formation of condensation inside the nasal mask.

\section{Temperature}

If the air from CPAP is too cold, you can warm up more the air in the bedroom. If this is not enough, you can use a humidifier/heater.

\section{Nasal allergies}

Sometimes allergies can be increased by use of nasal CPAP. Also allergenic factors, if present in the environment, should be removed with an air purifier. CPAP should be kept off the ground. The filters should be changed frequently and very thin additional filters can be added to the circuit. Humidifiers should be used only with demineralized water (not distilled). The use of antihistamines or topical steroids via a nasal spray can be useful.

\section{Nasal dryness}

Nasal wash with saline is effective at bedtime.

\section{Nasal congestion}

You should check for the presence of turbinate hypertrophy and allergies: cyclic use of steroids (nasal sprays or, if less important, nasal patches that facilitate opening of the wings of the nose).

\section{Irritation of nasal mucosa}

You must use a humidifier/heater, or adjust the system in use. Topical medications, such as oil or Vaseline $^{\circledR}$, are useful.

\section{Mask pressure}

The mask should not be too narrow. You must choose the right type that fits perfectly to the face and prevents loss of air. You can choose various types and models. Attention must be paid to signs of nasal irritation or ulceration of the nasal skin: you must use appropriate spacers to reduce the pressure of the mask on the nose. Where appropriate, consider using special nasal masks.

\section{Skin allergies}

They are often caused by detergents used to clean the mask. You must rinse thoroughly after use of detergents. Frequent change of cleaning products is recommended.

Figure 5 Most common problems in CPAP use.

Abbreviation: CPAP, continuous positive airway pressure.

Sleepiness Scale was $14 \pm 4$ ) and 32 were lost to follow-up. The average hours of CPAP use at three months were 7.3 hours per night, seven hours per night at one year, and five hours per night at two years. Daytime sleepiness measured by the Epworth Sleepiness Scale was $6 \pm 4$ at three months and $5 \pm 4$ at one and two years. The degree of patient satisfaction was reported as follows: $60 \%$ of patients felt very satisfied with CPAP, $32 \%$ were sufficiently satisfied, and $8 \%$ were poorly satisfied at three months. At one year, $65 \%$ of patients felt very satisfied,
$28 \%$ were sufficiently satisfied, and $7 \%$ were poorly satisfied. At two years, $49 \%$ of patients felt very satisfied, $3 \%$ were sufficiently satisfied, and $48 \%$ were poorly satisfied. To assess the causes of noncompliance with ventilation, we evaluated the difference between basic sleepiness measured by the Epworth Sleepiness Scale and basal AHI in subjects who spontaneously suspended CPAP and patients who had continued the treatment. We did not find any statistically significant difference between the two groups. 


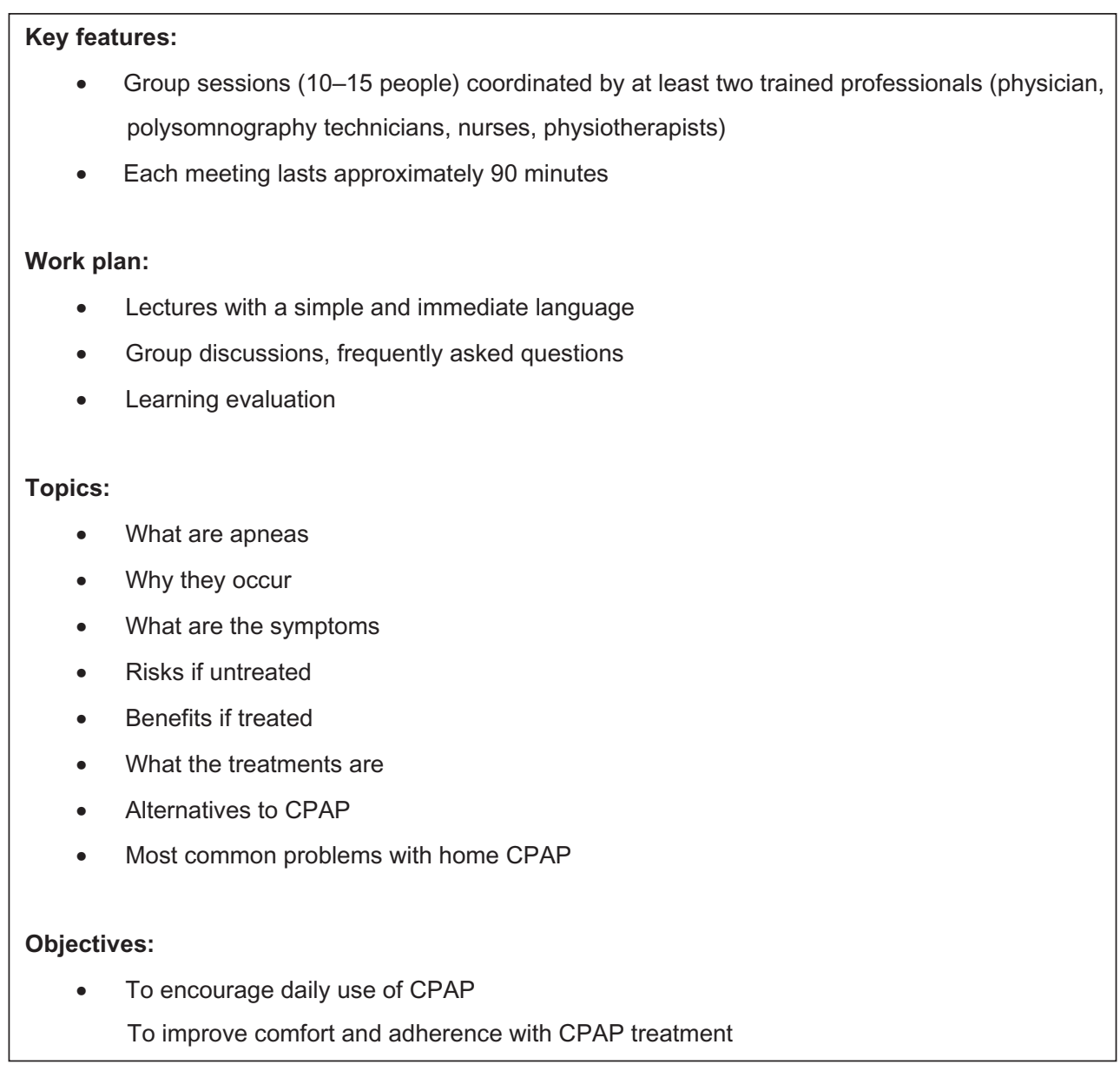

Figure 6 Educational program for home CPAP therapy in the long term.

Abbreviation: CPAP, continuous positive airway pressure.

\section{Discussion}

We investigated the effectiveness and importance of an educational protocol on adherence to CPAP in the short term (three months) and long term (one and two years) after the beginning of night ventilation. Our data seem to show the effectiveness of our educational protocol on appropriate adherence to ventilation at three months and one year of CPAP treatment. This result seems to be linked to the educational program performed in intensive mode during hospitalization and to the long-term support program at three months and one year after discharge from hospital. At two years, one year after suspension of our program, adherence to CPAP was reduced in our patients.

The aim of this study was to test a method of organizing and applying educational support for long-term night ventilation in patients with obstructive sleep apnea syndrome, in order to verify if a more efficient and reliable follow-up system could prolong CPAP use. One of the potential criticisms of this study is that it is a methodological protocol to increase CPAP compliance in obstructive sleep apnea syndrome patients, therefore does not take into consideration reasons why patients stopped using CPAP or psychological variables to enhance CPAP compliance.

Various types of CPAP machines are available, and can monitor the effectiveness of treatment, losses from the mask, persistence of snoring, and residual apneas/hypopneas. These models call our attention to recognizing these parameters and correcting them if possible. This supporting system seems to be closely linked to the success of adherence with ventilation. ${ }^{22}$ Intervention cannot be based only on solving technical problems, but must be related to socioeconomic and psychological factors, which may affect the ability of the patient to manage his/her health problems and are closely linked with daily use of CPAP. ${ }^{23,24}$

CPAP therapy shows its effectiveness for most patients, but its costs are high. According to this discussion, more restrictive criteria in the US are required for reimbursement of CPAP, such as demonstrated adherence with ventilation. ${ }^{14}$ 
The definition of adherence with minimum ventilation on a nightly basis is an even more complex issue. Several studies have demonstrated a need for daily use of CPAP for at least 4-6 hours per night to have an improvement in symptoms and daytime sleepiness. ${ }^{25}$ It is still rather unclear how many hours of night use are needed to reduce cardiovascular risk. Campos-Rodriguez et $\mathrm{al}^{26}$ have shown that those patients who use CPAP for at least $2-5$ hours every night have a reduced risk of mortality and morbidity compared with those who use it one hour per night.

Intensive adaptive training appeared to provide good adherence in the short term (three months), reaching compliance levels in excess of $80 \%$, associated with an average use of more than seven hours per night and with high levels of subjective satisfaction reported by patients. These results seem to coincide with the literature. Budhiraja et $\mathrm{al}^{3}$ have shown that the first 3-7 days of treatment are closely linked with the subsequent use of the CPAP ventilator after 30 days.

McArdle et $\mathrm{al}^{27}$ reported that $68 \%$ of patients continued treatment at five years. In fact, long-term compliance with night ventilation is unlikely and is linked to the support system of the center. Therefore, our long-term support program, running for three months and one year, could achieve good compliance, even over a long period, with $80 \%$ adherence and daily use of the ventilator for at least seven hours per night at one year. Without our educational support program, we could see a sharp drop in adherence during the second year. The initial experience and educational reinforcement seemed to play an important role on early adherence to ventilation, but this effect seems to be lost in the long term, suggesting the need to continue the motivational support.

It must be stressed that our program was designed mainly for a rehabilitation facility, but its intent was to open up discussion with all the organizations that deal with cardiorespiratory sleep disorders. In an increasingly cost-conscious environment, costs can be minimized by training of specialized teams to manage groups of patients with whom they stay in contact also by periodic telephone calls. We are aware that our study has some limitations. We considered a mainly male population referred to a single medical center. It was not possible to identify reasons why some patients had abandoned the program. The study population presented with moderate to severe AHI during sleep and, it is known that the severity of the apneic framework is one of the factors affecting compliance with ventilation. Importantly, this was a retrospective study and did not include a control group in the protocol.

\section{Acknowledgment}

The authors appreciate the language support provided by Dr Sabrina Dal Pra.

\section{Disclosure}

The authors report no conflicts of interest in this work.

\section{References}

1. Sullivan CE, Issa FG. Obstructive sleep apnea. Clin Chest Med. 1985;6(4):633-650.

2. Hudgel DW. Variable site of airway narrowing among OSAS patients. J Applied Physiol. 1986;61(4):1402-1409.

3. Budhiraja R, Quan SF. Sleep-disordered breathing and cardiovasular health. Curr Opin Pulm Med. 2005;11(6):501-506.

4. Barbe F, Pericas J, Munoz A, et al. Automobile accidents in patients with sleep apnea syndrome. An epidemiological and mechanicistic study. Am J Respir Crit Care Med. 1998;158(1):18-22.

5. Kapur VK, Redline S, Nieto FJ, et al. The relationship between chronically disrupted sleep and healthcare use. Sleep. 2002;25(3): 289-296.

6. Munoz A, Mayoralas LR, Barbe F, et al. Long-term effects of CPAP on daytime functioning in patients with sleep apnoea syndrome. Eur Respir J. 2000;15(4):676-681.

7. Engleman HM, Martin SE, Deary IJ, et al. Effect of continuous positive airway pressure treatment on daytime function in sleep apnoea/ hypopnoea syndrome. Lancet. 1994;343(8897):572-575.

8. Papperell JC, Ramdassingh-Dow S, Costhwaite N, et al. Ambulatory blood pressure after therapeutic and subtherapeutic nasal continuous positive air way pressure for obstructive sleep apnoea: a randomised parallel trial. Lancet. 2002:359(9302);204-210.

9. Marti S, Sampol G, Munoz X, et al. Mortality in severe sleep apnoea/ hypopnoea syndrome patients: impact of treatment. Eur Respir J. 2002;20(6):1511-1518.

10. Engleman HM, kingshott RN, Wraith PK, et al. Randomized placebocontrolled crossover trial of continuous positive air way pressure for mild sleep apnoea/hypopnea syndrome. Am J Respir Crit Care Med. 1999;159(2):461-467.

11. Smith I, Nading V, Lasserson TJ. Educational, supportive and behavioral interventions to improve usage of continuous positive airway pressure machines for adults with obstructive sleep apnoea. Chocrane Database Syst Rev. 2009;2:CD007736.

12. Meurice JC, Dore P, Paquerau J, et al. Predictive factors of long-term compliance with nasal continuous positive airway pressure treatment in sleep apnea syndrome. Chest. 1994;105(2):429-433.

13. Kribbs NB, Pack Al, Kline LR, et al. Effects of one night without nasal CPAP treatment on sleep and sleepiness in patients with obstructive sleep apnea syndrome. Am Rev Respir Dis. 1993;147(5): 1162-1168.

14. Aloia S, Knoepke C, Lee-Chiong T. The new local coverage determination criteria for adherence to positive airway pressure treatment: testing the limits? Chest. 2010;138(4):875-879.

15. Pieters TH, Collard PH, Aubert G, Dury M, Delguste P, Rodenstein DO. Acceptance and long term compliance with nCPAP in patients with obstructive sleep apnea syndrome. Eur Respir J. 1996;9(5): 939-944.

16. Chervin RD, Theut S, Bassetti C, Aldrich MS. Compliance with nasal CPAP can be improved by simple interventions. Sleep. 1997;20(4): 284-289.

17. Fletcher FC, Luckett RA. The effect of positive reinforcement on hourly compliance in nasal continuous positive airway pressure users with obstructive sleep apnea. Am Rev Respir Dis. 1991;143(5 Pt 1): 936-941.

18. Chervin RD, Theu S, Bassetti C, Aldrich MS. Interventions to improve compliance with nasal CPAP. Sleep Res. 1996;25:220-226. 
19. Likar LL, Panciera TM, Erickson AD, Rounts S. Group education sessions and compliance with nasal CPAP therapy. Chest. 1997;111(5):1273-1277.

20. [No authors listed]. Sleep-related breathing disorders in adults: recommendations for syndrome definition and measurement technique in clinical research. The report of an American Academy of Sleep Medicine Task Force. Sleep. 1999;22(5):667-689.

21. Rota M, Zibetti S, Pirola A, Patruno V, Aiolfi S. Adherence to CPAP treatment in patients with obstructive apnea during sleep. The role of respiratory rehabilitation therapist. ARIR. 2001;10(1):20-24.

22. Weaver TE, Kribbs NB, Pack AI, et al. Night-to-night variability in CPAP use over first three month of treatment. Sleep. 1997;20(4): 278-283.

23. Platt AB, Field SH, Asch DA, et al. Neighborhood of residence is associated with daily adherence to CPAP therapy. Sleep. 2009;32(6): 799-806.
24. Simon-Tuval T, Reuveni H, Greenberg-Dotan S, et al. Low socioeconomic status is a risk factor for CPAP adherence among adult OSAS patients requiring treatment. Sleep. 2009;32(4):545-552

25. Bennett SL, Barbour C, Langford B, et al. Health status in obstructive sleep apnea. Relation with sleep fragmentation and daytime sleepiness, and effects of continuous positive airway pressure treatment. Am J Respir Crit Care Med. 1999:159(6):1884-1890.

26. Campos-Rodriguez F, Perez-Ronchel J, Grilo-Reina A, Lima-Alvarez J, Beneitez MA, Almeida-Gonzalez C. Long-term effect of continuous positive airway pressure on BP in patients with hypertension and sleep apnea. Chest. 2007;132(6):1847-1852.

27. McArdle N, Devereux G, Heidarnejad H, et al. Long-term use of CPAP therapy for sleep apnea/hypopnea syndrome. Am J Respir Crit Care Med. 1999;159(4 Pt 1):1108-1114.

\section{Publish your work in this journal}

Patient Preference and Adherence is an international, peer-reviewed, open access journal focusing on the growing importance of patient preference and adherence throughout the therapeutic continuum. Patient satisfaction, acceptability, quality of life, compliance, persistence and their role in developing new therapeutic modalities and compounds to optimize clinical outcomes for existing disease states are major areas of interest. This journal has been accepted for indexing on PubMed Central. The manuscript management system is completely online and includes a very quick and fair peer-review system. Visit http://www.dovepress. com/testimonials.php to read real quotes from published authors. 